\title{
LA DIGITALIZACIÓN DEL PATRIARCADO: RETENCIÓN DEL TALENTO FEMENINO EN LAS EMPRESAS TECNOLÓGICAS
}

\author{
PATRIARCHY DIGITALIZATION: FEMALE TALENT RETENTION IN ICT
}

\author{
Laura Martínez Álvaro \\ Universidad Complutense de Madrid
}

\section{RESUMEN}

En una sociedad cada vez más tecnológica, las mujeres continúan infrarrepresentadas en el sector TIC y, muy especialmente, en los puestos de toma de decisión. La investigación se centra en identificar las barreras subyacentes a la presencia femenina en los entornos tecnológicos corporativos a través del análisis del marco teórico existente y, especialmente, desde el punto de vista de las autoras de referencia: Rosabeth M. Kanter, desde la teoría de comportamiento organizacional, y Judy Wajcman, desde la teoría feminista. El estudio se complementa con un trabajo de campo, todavía en curso, donde se contextualizan los resultados con la realidad del sector TIC en España a través de un análisis cuantitativo y cualitativo centrado en entrevistas en profundidad a una muestra representativa de directivos/as del sector. Como conclusiones preliminares, se observa una digitalización del patriarcado y la necesidad de abordar las intervenciones de forma estructural.

Palabras clave: tecnología, género, STEM, empleo, digital, retención.

\section{ABSTRACT}

In an increasingly technological society, women remain underrepresented in the ICT sector and, especially, in decision-making positions. The research focuses on identifying the underlying barriers to female presence in technological corporations through the analysis of the existing theoretical framework and, especially, from the point of view of the reference authors: Rosabeth M. Kanter, from the theory of organizational behavior perspective, and Judy Wajcman, from the feminist theory perspective. The study is complemented by a field work, still ongoing, where the results are contextualized with the reality of the Spanish ICT sector through a quantitative and qualitative analysis focused on in-depth interviews with a representative sample of ICT managers. As preliminary conclusions, we observe the digitization of patriarchy and the need to address interventions in a structural way.

Keywords: ICT, Gender, STEM, Employment, Digital, Retention. 


\section{Introducción}

Los avances tecnológicos de los últimos tiempos han supuesto una cuarta revolución industrial marcada por la convergencia de tecnologías digitales, físicas y biológicas que está cambiando el mundo a gran escala y a toda velocidad. El Internet del Todo (Internet of Everything) permite que personas, procesos, objetos y datos estén conectados las 24 horas los 365 días del año. Dicha transformación digital aumentará de forma significativa y tendrá una importancia mayúscula en la forma de entender el mundo que nos rodea y los avances de la humanidad. La tecnología es y será, en mayor medida, un importante motor de empleo.

En este contexto, la participación de las mujeres es decisiva a la hora de visibilizar y priorizar los problemas existentes y sus posibles soluciones en esta sociedad del conocimiento. Existe evidencia empírica que demuestra cómo la marginación de las mujeres de la comunidad tecnológica ha influido en el diseño, el contenido técnico y el uso de artefactos como el microondas (Cockburn y Ormrod, 1993), el teléfono (Martin, 1991), la robótica y el software (Suchman, 2008). Si bien la investigación destaca los fines sociales, cabe mencionar que, desde un punto de vista empresarial, existen diversos estudios que muestran cómo una organización más paritaria tiene unos mejores resultados de negocio (Catalyst, 2004; McKinsey, 2011), así como una mayor capacidad de innovación y creatividad (Tyson, 2003). No obstante, las mujeres aparecen infrarrepresentadas en el sector TIC, tanto a nivel horizontal como vertical, de forma crónica.

\subsection{Contexto laboral, género y Tecnologías de la Información y Comunicación (TIC)}

Las mujeres tienen una representación inferior a los hombres en el tejido de empresas tecnológicas españolas y un papel muy reducido en los puestos de toma de decisión. En 2014, un análisis de la distribución por sexo del número de afiliados y afiliadas al sistema de la Seguridad Social' en el sector de servicios informáticos avanzados ${ }^{2}$ constata que éste es un sector eminentemente masculino. A partir de los datos de 2014 de la Tesorería General de la Seguridad Social (TGSS), la Figura 1 muestra gráficamente cómo los hombres alcanzan el $68,33 \%$ de la afiliación frente al $31,67 \%$ de las mujeres en este sector. En cambio, la afiliación total para hombres y mujeres en España está más equiparada $(53,38 \%$ y $46,62 \%$

1 Estudio Prospectivo del Sector de Servicios Avanzados a las Empresas en las Tecnologías de la Información y Comunicación, Servicio Público de Empleo Estatal, 2015.

2 Servicios informáticos avanzados son aquellos servicios incluidos en los epígrafes de actividades económicas de la CNAE 2009 que se describen a continuación: 62. Programación, consultoría y otras actividades relacionadas con la informática y 63 . Servicios 
respectivamente) concluyendo que la representación de las mujeres en servicios informáticos avanzados es quince puntos porcentuales más baja que en el total de afiliados y afiliadas en España. Respecto a la contratación y el desempleo, el mismo estudio recoge una cuota de participación femenina sobre el total de contratos contabilizados de $38,48 \%$ y un $51,45 \%$ sobre el desempleo de larga duración en el sector TIC.

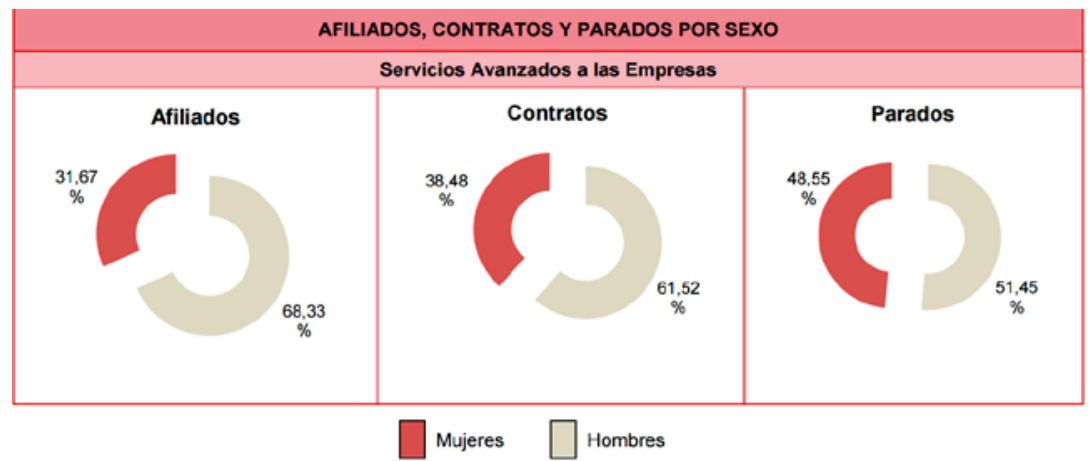

Figura 1. Afiliación, contratación y paro por sexo, TGSS 2014.

Dentro de la Unión Europea ${ }^{3}$, los datos no son diferentes ya que las mujeres aparecen infrarrepresentadas entre los especialistas TIC en todos los Estados miembros (Figura 2) donde los hombres representan un $80 \%$ de los especialistas. Cabe destacar el contraste entre la proporción de hombres y mujeres en empleos TIC con los hombres y mujeres en el empleo total, donde aparecen más igualados/as (53,9\% de hombres y $46,1 \%$ de mujeres).

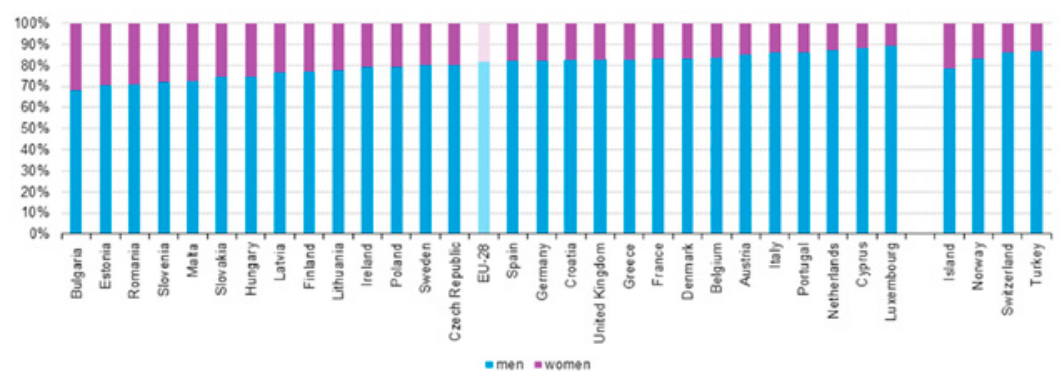

Figura 2. Especialistas TIC por género, Eurostat 2014. 
Además, solo un 19,2\% de los puestos de responsabilidad son ocupados por mujeres ${ }^{4}$ y se trata de puestos de carácter funcional (Finanzas, Marketing, Recursos Humanos, etc.) y no de aquellos puestos asociados a la estrategia de negocio o comercial, puestos desde donde se accede, de forma natural, a puestos de alta dirección. En Estados Unidos solo un $26 \%$ de los profesionales TIC son mujeres, la misma proporción que en 1960; el dato empeora para las ingenieras que representaban solo el $12 \%$ en $2013^{5}$.

En España, la evolución de la representación femenina entre los profesionales TIC no ha mejorado de forma significativa en los últimos años. Según datos del Instituto Nacional de Estadística ${ }^{6}$, se puede observar una representación relativamente estable en torno al $30 \%$ según se representa en la Figura 3:

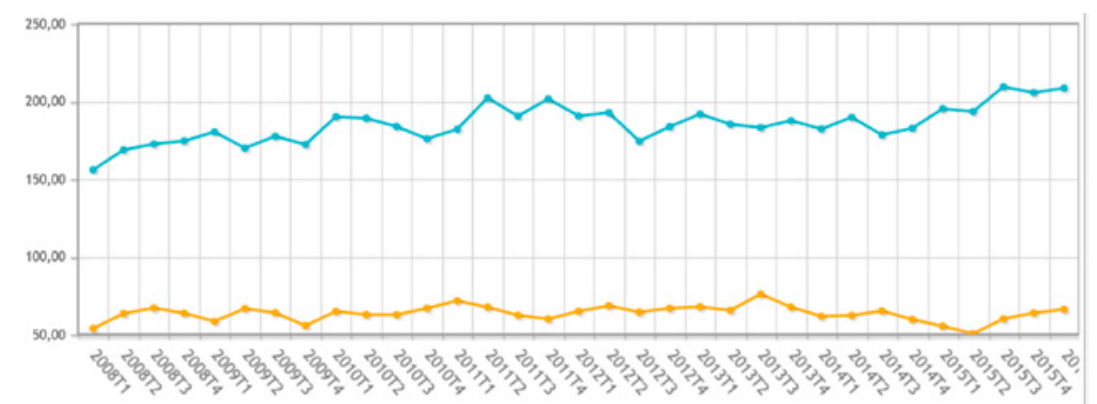

Figura 3. Evolución por género y rama de actividad TIC, 2008-2016, INE 2016.

Los resultados de una encuesta ${ }^{7}$ realizada en España a los profesionales TIC sobre las barreras que perciben para la escasa presencia femenina en su sector, señalan el techo de cristal como principal barrera, así como los salarios poco atractivos al inicio $191,89 \%$ y $89,81 \%$ respectivamente). Un $86 \%$ opina que existe una imagen masculina de la profesión y el peso de los estereotipos de género durante la educación secundaria también juega un papel importante $(76 \%)$, así como el hecho de que haya poca información de casos de éxito de mujeres $(88 \%)$.

4 Women active in the ICT sector, Comisión Europea, Directorate-General for Communications Networks, Content and Technology, 2013

5 Solving the Equation: The Variables for Women's Success in Engineering and Computing, 2015.

6 Encuesta de Población Activa para población ocupada en Programación, consultoría y otras actividades relacionadas con la informática (de acuerdo con la Clasificación Nacional de Actividades Económicas 62. Programación, consultoría y otras actividades relacionadas con la informática y 63.Servicios de información), valor absoluto, por sexo. INE 2016.

7 Encuesta «Opinión de profesionales de TIC sobre la escasa presencia femenina en el empleo tecnológico» realizada a partir de 1370 respuestas válidas a través de cuestionario on-line $(58,1 \%$ hombres y $41,9 \%$ mujeres) de personas que trabajan o desarrollan su actividad en España (98,5\%). Asociación de Técnicos de Informática (ATI) (European Centre for Women and Technology), 2013.

Dossiers Feministes, 22, 2017, 29-48 - ISSN: $1139-1219$ - DOI: http://dx.doi.org/10.6035/Dossiers.2017.22.3 
A continuación, la Figura 4 refleja el total de los factores y opiniones recogidas entre los y las participantes:

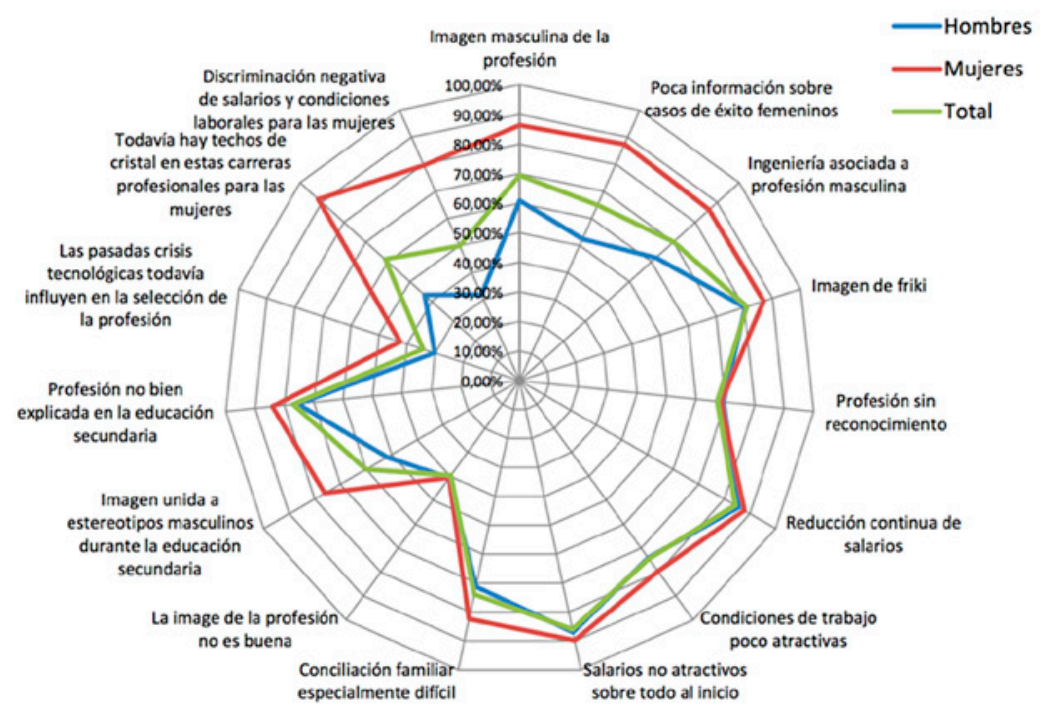

Figura 4. Factores que influyen en la baja presencia femenina en TIC, ATI 2013.

Por el contrario, una encuesta ${ }^{8}$ realizada a 246 empresas del sector TIC en España señala, como causa principal, la escasez de profesionales en las ocupaciones señaladas: la falta de especialización para realizar los cometidos requeridos por las empresas $(28,44 \%)$, la carencia de experiencia $(25,93 \%)$, así como la insuficiente formación $(19,11 \%)$ para desempeñar las funciones del puesto.

\subsection{Teorías feministas sobre la relación de las mujeres con la tecnología}

Inicialmente las teorías feministas mostraron un profundo escepticismo ante la masculinidad inherente a la tecnología y cómo ésta se había acentuado con conceptos dicotómicos y antagónicos de tipo la razón/la emoción o lo artificial/lo natural para caracterizarla (Harding, 1986). Una extensión de la idea de asociar las máquinas a lo

8 El cuestionario fue contestado por 246 empresas que se pueden agrupar de acuerdo con la Clasificación Nacional de Actividades Económicas (CNAE-2009) en su mayoría del grupo 62. Programación, consultoría y otras actividades relacionadas con la informática y 63 . Servicios de información $(65,85 \%$ y $6,91 \%$ respectivamente). 
masculino desde la prehistoria, cuando los seres humanos empiezan a elaborar sus propias herramientas (Castaño, 2005). Cabe destacar la existencia de múltiples investigaciones que han demostrado que la exclusión de las mujeres de la tecnología ha sido una consecuencia de la especialización de los oficios por hombres durante la revolución industrial (Bradley, 1989; Cockburn, 1983; Milkman, 1987). Esta tendencia fue remplazada a principios de los noventa del siglo XX por un optimismo inesperado acerca del potencial liberador de la «tecnociencia» para las mujeres y su poder para transformar las relaciones de género (Castells, 1996; Kemp y Squires, 1998; Green and Adam, 2001). Las ciberfeministas describen la era digital como un espacio donde las diferencias sexuales se diluyen, donde las mentes se liberan de sus cuerpos, donde su identidad no está sujeta ni determinada por su físico (Millar, 1998; Plant, 1998). Surge la metáfora del cyborg - ser que no es ni humano ni máquina, ni hombre ni mujer - como la posibilidad de transformar las relaciones entre las mujeres y las tecnologías (Haraway, 1997) o el ciberfeminismo de Sadie Plant en su obra «Ceros y Unos» como reacción al sistema patriarcal en las relaciones tecnológicas. La socióloga feminista Judy Wajcman manifestó la ingenuidad inicial del movimiento feminista en relación a la tecnología y las posibilidades que ofrecía un mundo sin identidades asignadas, neutro y por construir (Wajcman, 2004). En esta misma línea, Wendy Faulkner afirma que «la tecnología ha sido socialmente construida, o coproducida, junto con el género» (Faulkner, 2001: 79).

\subsection{Incorporación de las mujeres al mundo corporativo}

La incorporación de las mujeres al mundo corporativo está ligada a la máquina de escribir, inventada a finales del siglo XIX. En 1880, las mujeres representaban un $4 \%$ del trabajo administrativo, es decir, de las tareas asociadas con mecanografía, registro, contabilidad, etc. Diez años más tarde, en 1890, su participación ascendía a un $21 \%$ de dichas tareas administrativas. En 1920, las mujeres ocupaban el $91.8 \%$ de los puestos de mecanografía y un $48.8 \%$ de los puestos de contabilidad, registro y caja ${ }^{9}$. De esta forma, las tareas administrativas -que habían sido ejercidas mayoritariamente por hombres- se feminizaron. Si bien las escuelas de mecanografía estaban abiertas a mujeres y hombres, las clases incluían aspectos de imagen personal, manicura, vestimenta y conducta esperada (Kanter, 1977). Las corporaciones modernas iban evolucionando con la incorporación de nuevas funciones y nuevos roles; junto con la figura del manager (gerente), surgen los roles de secretaria y esposa de manager retratados por Kanter en su obra Men and Women of the Corporation de 1977.

9 Los cálculos están basado en los datos de Hill, Joseph «Women in Gainful Occupations 1870-1920» y Margery Davis «Woman's Place Is at the Typewriter: The Feminization of the Clerical Labor Force», Radical America, 8 (July-August 1974) (Kanter, 1977). 
Durante la mayor parte del siglo XX, impera en las corporaciones una «ética masculina» dominada por el espíritu de racionalidad y contrapuesto con la emoción asociada a lo femenino. Esta asunción implicará en los años venideros, y hasta nuestros días, una creciente presencia de las mujeres en puestos relacionados con las personas y la comunicación (Lynch, 1973). Precisamente, esta asociación de mujer-emoción ha apartado a las mujeres de los puestos de toma de decisión hacia puestos más funcionales y administrativos que se han visto feminizados, mientras que la gestión de manager se ha masculinizado.

\section{Líneas de investigación}

El punto de partida y justificación de la investigación realizada se centra en determinar qué factores ralentizan la representación de las mujeres en el sector TIC, así como su participación en la toma de decisiones de las empresas tecnológicas españolas, dando por sentado que una representación más paritaria redefiniría las prioridades y necesidades actuales de la sociedad de forma más efectiva. Para ello, la investigación se basa en las siguientes líneas de análisis:

- Analizar comparativamente las investigaciones realizadas por Rosabeth Moss Kanter en EEUU (1977) y Judy Wajcman en Reino Unido (1998) en relación a la situación de las mujeres en las corporaciones: ¿qqué barreras existen de carácter aparente y qué barreras existen de carácter profundo?,

- Analizar cualitativamente el empleo femenino en el contexto de las corporaciones tecnológicas españolas e,

- Identificar las claves de las barreras reales que perciben las mujeres para alcanzar puestos de responsabilidad en el sector TIC.

La investigación se centra en los trabajos que realizaron las académicas sociólogas Rosebeth Moss Kanter y Judy Wajcman, la primera a finales de los años 70 y la segunda a finales de los años 90 del siglo XX. Ambas académicas centraron sus estudios en el empleo, así como en el entorno de trabajo de las corporaciones de cultura anglosajona, si bien desde perspectivas diferentes. Kanter utiliza un marco conceptual basado en las teorías del comportamiento organizacional, mientras que Wajcman centra sus investigaciones en el marco de la teoría feminista. Ambas resultan complementarias a la vez que continuistas en sus hallazgos. Cabe destacar dos aspectos fundamentales por los que son las académicas elegidas para la realización de este análisis comparativo: un primer aspecto se centra en el enfoque de igualdad entre trabajadores y trabajadoras, es decir, las autoras no se centran 
en ningún momento en diferenciar los estilos, características o aptitudes de los trabajadores y trabajadoras por su género. En este sentido, ambas manifiestan su interés en obviar las diferencias que se atribuyen a las mujeres, por el hecho de ser mujeres, diferencias que no entran a valorar. El segundo aspecto se centra en que ambas autoras buscan factores y barreras culturales inherentes a la organización, aquellas de carácter estructural e institucional y, no aquellas que forman parte de las personas de la organización.

Estos dos aspectos constituyen el eje predominante de la presente investigación, es decir, se analiza un escenario donde se parte de la igualdad de las personas en cuanto a aptitudes, actitudes, capacidad de trabajo, conocimiento, etc. y centrado en los factores de naturaleza organizacional. Tal y como manifestaba Crozier, todas las personas de la organización son racionales, independientemente de lo apropiadas o no de sus manifestaciones o comportamientos que, por otra parte, suelen ser el resultado de su particular situación en la organización con el fin de preservar su dignidad, control y reconocimiento (Crozier, 1964).

\subsection{La cuestión del liderazgo femenino}

Existe abundante literatura científica y divulgativa centrada en las diferencias de estilos de liderazgo corporativo en función del género asignado; estos estudios otorgan a las mujeres una mayor capacidad de escucha, de comunicación, de empatía y de consenso que los hombres, pero también menor seguridad, confianza, riesgo, ímpetu, que sus compañeros. Recuerda, salvando las distancias, al feminismo de la diferencia que surge como pensamiento político reivindicativo a finales de los 60 del siglo pasado. Un feminismo basado en la exaltación de las cualidades femeninas frente a las masculinas y alejado de una sociedad formada por individuos más allá de los géneros asignados. La pensadora Betty Friedan señala los obstáculos que han supuesto para la participación de la mujer en la sociedad esas cualidades femeninas atribuidas (Friedan, 1963). La filósofa Celia Amorós insiste en su obra en que esa diferencia de identidad no existiría si no hubiera habido un sistema de patriarcado de dominación (Amorós, 1994).

En los últimos años, ha surgido el movimiento corporativo Lean In de la mano de la directiva ejecutiva de Facebook, Sheryl Sandberg. Este movimiento justifica la falta de presencia femenina en el sector tecnológico por las limitaciones de tipo cultural autoimpuestas por las propias mujeres. La autora insta a las mujeres del mundo corporativo a ser más ambiciosas, a involucrarse más en las decisiones y asumir retos nuevos (Sandberg, 2013). En definitiva, la igualdad efectiva es una carta que debe jugar cada mujer desde su propio territorio. Este movimiento cuenta con muchos adeptos y adeptas que han centrado la problemática de la igualdad de oportunidades en las mujeres y no en la organización. Fruto 
de este razonamiento, las organizaciones más sensibilizadas con la igualdad, han lanzado programas dirigidos exclusivamente a mujeres con el objetivo de suplir estas supuestas carencias a través de charlas motivacionales, formación de liderazgo femenino, iniciativas de mentoring, redes internas de mujeres, entre otros. En este sentido, la segregación entre hombres y mujeres en este tipo de programas, lejos de fomentar la igualdad de oportunidades, contribuye a fomentar las diferencias. De hecho, en palabras de la académica Wajcman, «la construcción de las mujeres como diferentes de los hombres (tomados como el estándar) es uno de los mecanismos por los que se mantiene el poder masculino» (Wajcman, 1998: 23).

Por otra parte, existen experimentos científicos que muestran cómo características habitualmente atribuidas a las mujeres en el trabajo (falta de ambición o motivación, intereses fuera del ámbito profesional, etc.) son identificadas igualmente en varones sometidos a entornos donde las estructuras de poder y oportunidad les excluyen (Cohen, 1958; Kanter, 1977). Por último, destacar las últimas tendencias que centran la igualdad de oportunidades en la diversidad como acicate del talento. Lejos de disolver las diferencias, esta tendencia se centra en valorarlas. Gestionar las diferencias no implica cambiar el orden hegemónico establecido por el patriarcado, al revés, anima a que un número más diverso de personas puedan encajar en puestos convencionalmente estructurados. En definitiva, tal y como diría Kanter:

La efectividad de las mujeres líderes, como la de los hombres líderes, es una respuesta a las oportunidades de poder, tener una posición de favor en una estructura de poder. De esta forma, tanto hombres como mujeres ejercitan su autoridad de forma más productiva y con mejores resultados cuando tienen poder detrás (Kanter, 1977: 343).

\subsection{El estigma de la conciliación}

El contexto laboral de las mujeres trabajadoras es distinto al de los hombres trabajadores. El hecho de que no compartan el mismo contexto, tiene un impacto negativo en la igualdad de oportunidades entre empleados y empleadas de una empresa. Dentro de este contexto, las empresas se han sentido muy cómodas con el discurso de la carga que supone para las mujeres el cuidado de los/as hijos/as y de las tareas del hogar y, por ello, su falta de disponibilidad para acceder a puestos de toma de decisión. Un discurso que centra el origen de las diferencias en la división sexual del trabajo en la familia y no en aquellas que se dan en el entorno de trabajo, en el seno de la organización. Independientemente de su rol en la esfera pública, las mujeres sufren la sistemática estigmatización del rol asumido socialmente en la esfera privada, es decir, se les atribuyen las responsabilidades propias del hogar, así como el cuidado de los familiares dependientes. Esta estigmatización se incrementa en 
función de la edad de la mujer y su cercanía a dichos eventos vitales independientemente de la situación particular de cada mujer (Wajcman, 1998).

En este sentido, la aprobación de la Ley Orgánica 3/2007, de 22 de marzo, para la igualdad efectiva de mujeres y hombres recogía, entre otras, medidas destinadas a mejorar la conciliación familiar que, si bien se establecían para todos los trabajadores y trabajadoras, se acogieron a ellas en su gran mayoría las mujeres trabajadoras dando paso a una carrera con menos oportunidades, menos salario y menos horas trabajadas. Casi una década después, la presencia de las mujeres en el sector TIC y en puestos de dirección no se ha visto impactada de forma importante. Cabe pensar que las cuestiones de igualdad, y no las de conciliación, podrían ser más efectivas para aumentar la presencia de mujeres en puestos del sector (Castaño, 2010).

La investigación plantea identificar las barreras que encuentran las mujeres, más allá de las asociadas a la conciliación del trabajo doméstico y familiar. No hay duda de que es un factor importante e interrelacionado, pero se cuestiona hasta qué punto es decisivo en la falta de presencia femenina en el sector TIC.

\section{Metodología}

La metodología seleccionada para la investigación se basa en los mismos métodos de estudio empleados por las autoras de referencia (Kanter y Wajcman), desde un marco de referencia basado en los resultados del análisis comparativo entre ambas. Tras un primer análisis cuantitativo de las organizaciones analizadas, se recoge información, lo más desagregada posible, sobre la participación de la mujer y las características específicas de las organizaciones seleccionadas. Posteriormente, y todavía en curso, se realizará un análisis cualitativo con la inclusión de encuestas, grupos de discusión, entrevistas semi-estructuradas y de entrevistas en profundidad.

La observación, como herramienta de análisis cualitativo, ha sido descartada por la dificultad que entraña en un sector, de estas características donde la mayor parte de las decisiones se toman en reuniones y el día a día transcurre en proyectos de consultoría fuera de las oficinas.

\subsection{La entrevista en profundidad}

La entrevista en profundidad será la herramienta principal de investigación. Existen múltiples investigaciones de carácter cuantitativo y cualitativo en este ámbito de investiga- 
ción, no obstante, el análisis del discurso de las personas entrevistadas puede arrojar información relevante que, de otra forma, no sería posible rescatar. Una perspectiva de las personas entrevistadas donde la información se sitúa en un contexto multidimensional, atendiendo al entorno familiar, socioeconómico y cultural de la persona entrevistada; donde se analiza el significado de las palabras empleadas y se adentra en la intimidad con objeto de comprender la individualidad de la persona entrevistada. La entrevista en profundidad sigue el modelo de conversación entre iguales, "encuentros reiterados cara a cara entre el investigador y los informantes» (Taylor y Bogdan, 1975: 101), reuniones orientadas hacia la comprensión de las perspectivas que tienen los informantes respecto de sus vidas, experiencias o situaciones, tal como las expresan con sus propias palabras. Es importante crear un vínculo cercano, de confianza y sólido con las personas entrevistadas. Las entrevistas se realizarán fuera del entorno de trabajo y en un lugar donde la persona entrevistada se sienta cómoda. Las entrevistas serán encuentros programados y concluirán cuando se llegue a un punto de saturación donde ya no se incorpore nada nuevo a la información existente (Blasco y Otero, 2008).

El guión de la entrevista girará en torno a la hipótesis de la investigación y contendrá las temáticas a investigar, principalmente destacadas por las académicas de referencia: análisis de roles, estilos gerenciales, estereotipos de género, expectativas de carrera, relaciones de oportunidad y poder, sexo, diversidad y, por último, vida doméstica. El número de las entrevistas en profundidad dependerá del nivel de información que se obtenga, se iniciará realizando un par de entrevistas por cada uno de los perfiles ya delineados al principio de la investigación (Patton, 1990), y conforme se cubran las categorías de análisis del guión, se evaluará qué información hace falta todavía.

Las entrevistas se dividirán en dos fases: una de correspondencia (la base de la información es la conversación con la persona entrevistada) y una fase de análisis donde se trabajarán las conversaciones por categorías de análisis, las trascripciones y las codificaciones de las mismas. Las entrevistas serán grabadas con permiso de las personas entrevistadas y se empleará el software Atlas.ti para el tratamiento de las mismas ${ }^{10}$. Adicionalmente, y de forma complementaria, se realizarán entrevistas semi-estructuradas, así como grupos de discusión con grupos de interés que, aunque no son objeto directo del estudio, son personas cuyo trabajo se desempeña en las oficinas y tiene acceso a múltiples informaciones y situaciones relacionadas, en concreto, se trata de los puestos administrativos y de asistentes personales. Estas técnicas cualitativas de investigación serán complementadas por encuestas, de software libre, al mayor número de personas de los grupos profesionales objeto del estudio (de dirección, negocio y apoyo) en las organizaciones analizadas.

10 Se preservará la identidad de las personas entrevistadas bajo un pseudónimo, así como la privacidad y confidencialidad de las informaciones obtenidas. 


\subsection{Tipología de empresa}

Las investigaciones de dichas académicas se centraron en acotar el análisis a organizaciones con un componente tecnológico importante y de cultura anglosajona ${ }^{11}$, que cumplían con ciertos criterios: por una parte, un reconocido interés por estar a la última en políticas y mejores prácticas de igualdad de oportunidades; además debían ser empresas del sector privado, dado que contaba con un menor número de investigaciones que el sector público, y finalmente del sector TIC.

La presente investigación centra su análisis en las organizaciones que cumplen con estas características, destacando aquellas donde la atracción y retención del talento es un factor estratégico fundamental para la organización. Adicionalmente, se trata de empresas que forman parte del modelo flexible formalizado según la clasificación de culturas empresariales en la implementación de políticas de conciliación e igualdad (Castaño, 2010) donde se describe en torno a los siguientes elementos:

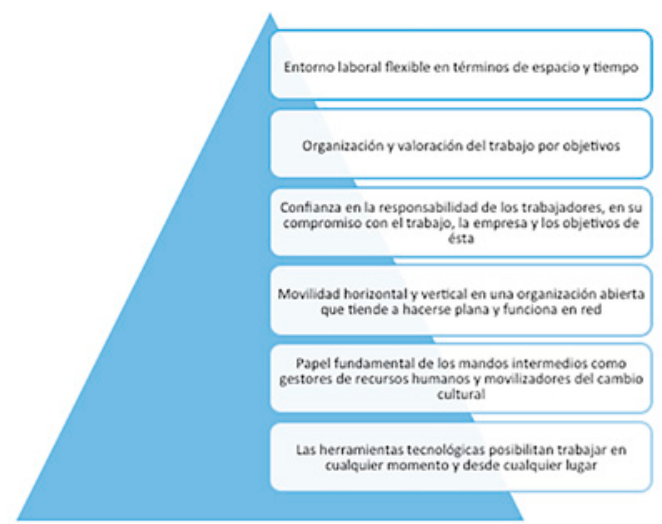

Figura 5. Elementos culturales del Modelo flexible formalizado/diversidad. Elaboración propia.

Las empresas que forman parte de este modelo consideran que la baja presencia de las mujeres constituye un problema para el negocio, entienden que el origen de esta baja representación está en el contexto sociocultural y en la elección de estudios por parte de las mujeres. No obstante, se trata de empresas que realizan actuaciones dirigidas a mejorar

11 En todos los casos, las organizaciones analizadas se mantuvieron en el anonimato. 
esta situación, bien a través de actuaciones de carácter interno y/o actuaciones en el ámbito universitario y centros escolares.

La empresa seleccionada para dicha investigación, es de origen anglosajón, con sus servicios centrales en Estados Unidos y con más de 350.000 empleados/as en el mundo. Establecida en España, cuenta con varias oficinas en el territorio y con una facturación anual entre 500 y 1.000 millones de euros. Cuenta con más de 8.000 empleados y empleadas, de los cuales más del $85 \%$ cuenta con titulación superior. Dicha organización, centra su negocio en los servicios de consultoría, así como los servicios de externalización. Actualmente, su Comité de Dirección está formado solo por hombres, su Comité Ejecutivo cuenta con un $9 \%$ de mujeres y un $25 \%$ en sus mandos intermedios. La compañía cuenta con un Plan de lgualdad y con diversos reconocimientos institucionales a nivel nacional e internacional. Las iniciativas de igualdad se lanzan desde los servicios centrales al resto de oficinas en el mundo y son complementadas, a nivel local, por el departamento de Recursos Humanos.

\subsection{Perfil de análisis}

La investigación incorpora la percepción de las personas de la organización en función de su formación y experiencia laboral, independientemente de su sexo y categoría profesional. Se ha considerado necesario analizar la percepción de las personas que ocupan otros puestos dentro de la empresa (por ejemplo, los puestos de apoyo), así como aquellos puestos de menor responsabilidad (asistente de dirección o administrativos) puesto que disponen de una perspectiva diferente, como observadores constantes, para identificar posibles sesgos de género en la organización. Es importante destacar que, en la corporación analizada, la selección de personas para puestos internos o de apoyo, se hace a partir de las personas que fueron seleccionadas inicialmente para puestos de negocio, es decir, las personas que han desarrollado su trayectoria profesional desde la línea de actividad principal y con mayor potencial de crecimiento. La representación femenina en esta categoría suele ser más elevada y, por ello, se consideran de interés para la investigación las personas que ocupan estas direcciones funcionales o de apoyo y su trayectoria profesional. Una adecuada estructura de la entrevista en profundidad requiere construir perfiles específicos de estudio ya que las características van a definir la estrategia de vinculación con la persona entrevistada.

El trabajo empleará unos criterios específicos de selección de las personas a entrevistar donde se consideren los siguientes aspectos: estudios universitarios, años de experiencia laboral, puesto ocupado, antigüedad en el cargo y antigüedad en la empresa. Cada uno de estos aspectos tendrá una horquilla que definirá su adecuación o no como sujeto investigado, como se muestra en la Figura 6. 


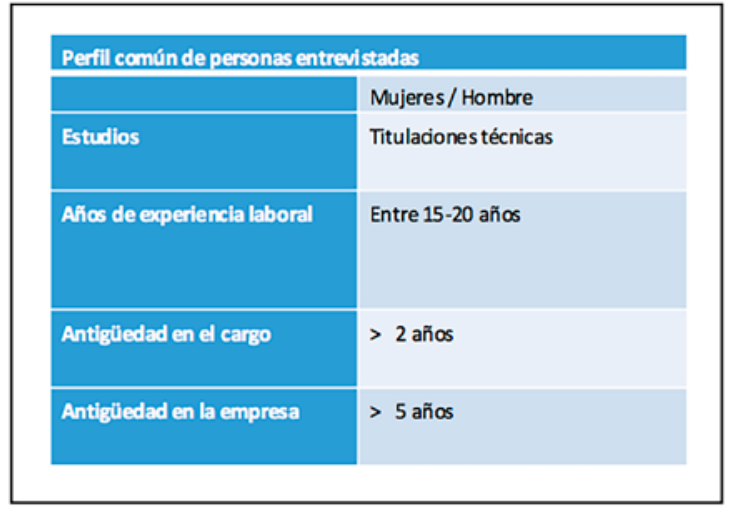

Figura 6. Requisitos necesarios para las entrevistas en profundidad. Elaboración propia.

Todas las personas entrevistadas, salvo las que formen parte de los grupos de discusión, deberán cumplir con estos requisitos de forma que el análisis pueda ser comparativo. Junto con estos requisitos, se entrevistará a personas que ocupen puestos de responsabilidad en distintos ámbitos de la empresa: profesionales de dirección, profesionales de negocio y profesionales de apoyo; para cada uno de estos grupos, se entrevistará a profesionales con hijos/as a cargo y sin hijos/as cargo en la misma proporción como se muestra la Figura 7.

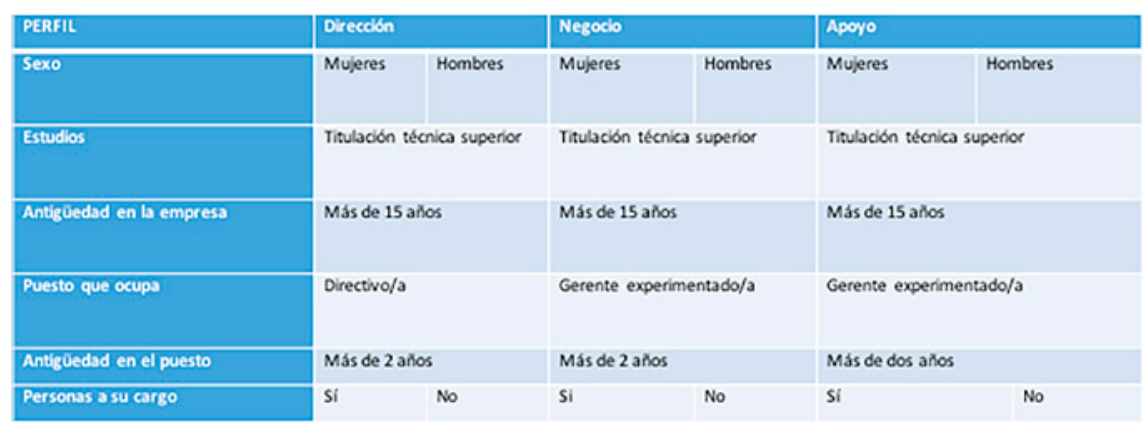

Figura 7. Criterios de selección de la muestra. Elaboración propia.

Inicialmente, se realizará un total de 24 entrevistas, con un número equitativo entre mujeres y hombres y con la misma proporción de personas a su cargo. Estas entrevistas serán de carácter semi-estructurado que servirá de filtro inicial para la selección de las personas para la entrevista en profundidad. 


\section{Resultados}

Si bien la investigación no ha concluido, cabe destacar unos primeros resultados de la misma para indagar acerca de las barreras aparentes y de aquellas más profundas que explican la escasa presencia de las mujeres en el sector TIC. Según las académicas estudiadas, las mujeres siguen siendo intrusas en una cultura laboral dominada por hombres. Un mundo corporativo que refleja las estructuras de poder del patriarcado, que concede privilegios a los hombres - en forma de oportunidades, poder, salario, tipo de contratación, participación en la toma de decisión, entre otras - e invisibiliza a las mujeres. Los tentáculos del patriarcado son muchos, existen varios factores que influyen y que pueden ser señalados: la gestión de oportunidades y poder, el tokenismo, la falta de masa crítica, los club de hombres, los sesgos de géneros implícitos $y$, por último, la cultura organizativa.

\subsection{Gestión de oportunidades y poder}

Las investigaciones de Kanter se centraron mucho en las relaciones de poder y oportunidad como continuación a los trabajos realizados por Crozier durante los años 60. Ambos académicos apuestan por la racionalidad implícita en las personas, que reaccionan en función de las presiones existentes en la organización para sobrevivir en su situación particular. En este sentido, los resultados de los experimentos llevados a cabo muestran que personas sin expectativas de promocionar o crecimiento, buscarán sus intereses fuera de la organización. Las oportunidades definen la motivación de las personas y la falta de ellas producirá unas reacciones a menudo atribuidas a las mujeres: falta de motivación, interés, ambición... De la misma forma, las personas expuestas al poder tendrán un comportamiento similar independientemente de su género. En este sentido, concluyeron que las mujeres en puestos de dirección tenían un comportamiento similar al de un hombre con poder.

\subsection{Club de hombres}

Se trata de un término anglosajón «clubbiness» acuñado para designar esa jerga, intereses y aficiones que comparten los hombres que forman parte de los centros de poder. Wajcman destaca en su investigación que las mujeres, especialmente aquellas en mandos intermedios, perciben este factor como una de las barreras más importantes en su progresión. En este sentido un $76 \%$ de las mujeres encuestadas los sitúan como uno de los factores principales, mientras solo un $43 \%$ de sus compañeros creen que pueda suponer una barrera para el progreso de sus compañeras. El British Institute of Management confirmó que dicho 
factor continuaba siendo la barrera más significativa para las mujeres (Charlesworth, 1997). Cabe destacar que la académica norteamericana Shere Hite tuvo la oportunidad de estudiar en profundidad la psique de algunos de los principales líderes de las corporaciones a finales de siglo XX. A través de su informe basado principalmente en entrevistas, describe los guetos femeninos dentro de las empresas en yuxtaposición al fenómeno de los clubs de hombres (Hite, 2000).

\subsection{Tokenismo/masa crítica}

El tokenismo, del inglés token, es la práctica de hacer un gesto superficial para la inclusión de las minorías. Debido a la falta de representación de mujeres en centros de poder, las mujeres que han conseguido situarse en éstos, sienten la presión de no ser tratadas como una persona más, sino representar su grupo social, en este sentido, «la mujer». Es una situación controvertida para ella puesto que será juzgada tanto si se comporta de acuerdo a «lo esperado socialmente» por el hecho de ser mujer, como si se comporta de una forma más parecida a los hombres. Independientemente de cuál sea su comportamiento, no será vista como una persona más sino en relación al grupo social al que pertenece generando una situación Catch-22 (Castaño, 2010)'12.

\subsection{Estereotipos de género}

Los estereotipos de género implícitos son un factor importante y difícil de gestionar. Existe una notable evidencia empírica que muestra cómo los estereotipos de género afectan a nuestra forma de juzgar y relacionarnos y cómo, en concreto, perjudican negativamente a las mujeres en el sector TIC. De hecho, la Universidad de Harvard desarrolló un programa informático que calcula, en función de la rapidez de respuesta del usuario, los estereotipos que mantiene el usuario respecto a las mujeres y las carreras técnicas ${ }^{13}$. Más del $75 \%$ de los más de un millón de usuarios que han participado en el test, han demostrado tener estos estereotipos de género. Estos estereotipos de género afectan en todas las fases de la carrera profesional de las mujeres: desde el colegio -a la hora de considerar su futuro profesionaldurante la carrera universitaria, en el proceso de selección de la empresa, en las evaluaciones de desempeño y finalmente en las promociones a puestos de mayor responsabilidad y

12 Las situaciones Catch-22 se producen cuando surge un dilema en la comunicación porque una primera respuesta positiva generará una respuesta que deniegue la primera respuesta.

13 https://implicit.harvard.edu 
compensación económica. Estudios científicos documentados ${ }^{14}$, para cada una de estas fases, reflejan la importante desventaja de las mujeres en el sector de la tecnología.

\subsection{Cultura organizativa}

La cultura organizativa no es un factor más ya que aúna los factores descritos anteriormente. La cultura organizativa tiene efecto en el comportamiento de las personas que forman parte de la organización, marcan el espíritu y directrices de la misma de una forma implícita y aceptada por los trabajadores y trabajadoras. Esta cultura es transmitida por las personas que ostentan el poder en la organización, son las personas que lanzan los mensajes necesarios con sus actos y con sus decisiones actuando como verdaderos gatekeepers guardianes de las esencias- de la organización. Este factor incide de forma crítica en la retención del talento. La cultura corporativa asumirá el comportamiento de estos gatekeepers como el modelo de liderazgo estándar y éste hecho afectará a los procesos de carrera, así como el ascenso a puestos de dirección (Webster, 2005). En este sentido, la cultura organizativa es garante del predominio masculino en las organizaciones.

\section{Conclusiones preliminares}

Si bien la investigación está en curso, a raíz de los resultados que se extraen de las revisiones teóricas de las autoras de referencia, podemos identificar como conclusión preliminar, la necesidad de realizar intervenciones de carácter estructural desde la organización que puedan transformar la cultura corporativa desde su base. La retención del talento femenino en las corporaciones tecnológicas continúa siendo un reto y las barreras profundas parecen inamovibles. Las estrategias implantadas para la retención del talento en el sector TIC han fracasado en términos generales. Las soluciones basadas en modelos de apoyo individual - como la formación, mentoring, permisos transferibles, entre otros -, deben dar paso a soluciones basadas en modelos de cambio estructural.

El problema no reside en los departamentos de Recursos Humanos o Responsabilidad Social Corporativa, el problema es inherente a la organización y, especialmente a su cultura organizativa. Los valores y creencias que propugna la organización son incompatibles con la supuesta estrategia de retención del talento femenino e igualdad de oportunidades. La cultura organizativa promueve una única medida, un único modelo, el de los hombres, como

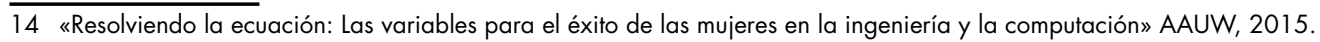


referencia de éxito en la organización. Las organizaciones que consideran el talento como factor estratégico tienen que dejarse permear no sólo por los valores de igualdad de oportunidades, si no ser proactivos para superar las barreras existentes para que el talento femenino se exprese, se desarrolle de forma exitosa y se quede en la organización. En este sentido, los valores deben ser incorporados a la misión de la organización, a su estrategia de negocio, a los programas y proyectos, a los presupuestos, a las campañas de marketing y comunicación, a la formación, a las relaciones con proveedores y con asociaciones, entre otros componentes, para que la estrategia de retención de talento sea efectiva dentro de la organización.

La Figura 8 recoge un esquema comparativo entre las características de las intervenciones basadas en modelos individuales y aquellas basadas en modelos estructurales.

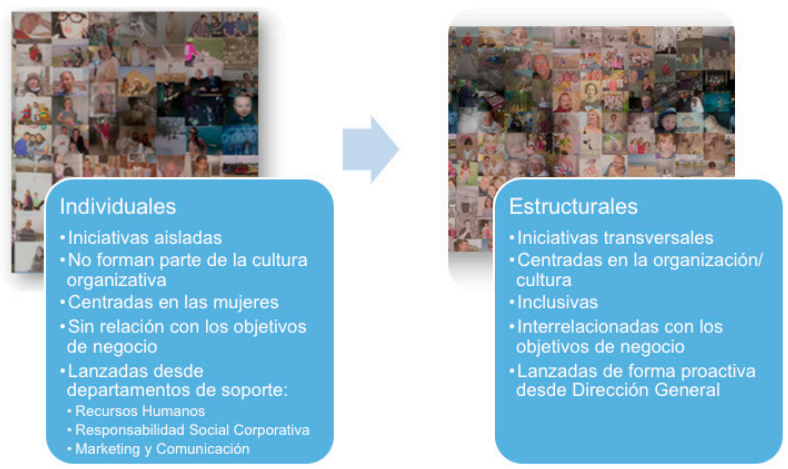

Figura 8. Modelos individuales vs. Modelos estructurales. Elaboración propia.

En definitiva, las estrategias de retención del talento femenino deberán plantearse desde un enfoque basado en modelos estructurales que permitan una transformación cultural en el conjunto de la organización de cara a eliminar las barreras profundas que subyacen como resultado de nuestra sociedad patriarcal. No se trata de una utopía, incrementar la presencia femenina en el sector $\mathrm{TIC}$ es posible, pero requiere un importante esfuerzo transformador desde la propia esencia de la organización. Los cambios organizativos son probablemente los más complejos pero los beneficios son incalculables, tanto para la organización, como para sus personas y la sociedad en general ya que, como apuntó Kanter, «si el trabajo hace a las personas, entonces las corporaciones son las productoras contemporáneas, por excelencia, de las personas» (Kanter, 1977: 3). 
Referencias bibliográficas

AMORós, Celia (1994): Espacio público, espacio privado y definiciones ideológicas de «lo masculino» y ilo femenino», México, UNAM.

BlAsco, Teresa y Otero, Laura (2008): Técnicas conversacionales para la recogida de dates en investigación cualitativa: La entrevista (I), NURE Investigación.

BRADLEY, Harriet (1989): Men's work, women's work: A sociological history of the division of labor in employment, Oxford, Polity Press.

CASTAÑO, Cecilia (2005): Las mujeres y las tecnologías de la información: Internet y la rama de nuestra vida, Madrid, Alianza Editorial.

CASTAÑO, Cecilia (2010): Género y TIC: Presencia, posición y políticas, Barcelona, UOC Ediciones. CASTELLS, Manuel (1996): The information age: economy, society and culture. Volume i: the rise of the network society, Cambridge, Blackwell.

ChARLESWORTH, K. (1997): A question of balance? A survey of managers' changing professional and personal roles, London, British Institute of Management Report.

COCKBURN, Cynthia (1983): Brothers: Male dominance and technological change, Londres, Pluto Press. CockBurn, Cynthia y OrmRod, Susan (1993): Gender and technology in the making, London, Sage.

COHEN, Arthur R. (1958): Upward communication in experimentally created hierarchies.» Human Relations, Vol 11, № 1.

Collinson, David; Collinson, Margaret y KNIGHTS, David (1990): Managing to discriminate (insurance industry), Londres y Nueva York, Routledge.

COBBETt, Christianne y HILL, Catherine (2015): Solving the Equation: The Variables for Women's Success in Engineering and Computing, Washington, AAUW.

Crozier, Michel (1964): El fenómeno burocrático, Buenos Aires, Amorrortu editores.

FAULKER, William (2001): The Technology Question in Feminism: A view from feminist technology studies, Women's Studies International Forum.

Friedan, Betty (1963): The Feminine Mystique, Neva York, W.W. Norton \& Company.

GluCSMANN, Miriam (1982): Women on the line. British Factory, Londres, Routledge.

Green, Eileen y Adam, Alison (2001): Virtual Gender: Technology, Consumption and Identity Matters, Londres, Routledge.

CaRAWAY, Donna Jeanne (1985): «A manifesto for cyborgs: science, technology and socialist feminism in the 1980', Socialist Review.» $N^{\circ} 80$.

CaraWaY, Donna Jeanne (1997): Feminism and Tecnoscience, Londres, Routledge.

Harding, Sandra G. (1986): The Science Question in Feminism, Cornell University Press.

Dossiers Feminists, 22, 2017, 29-48 - ISSN: $1139-1219$ - DOI: http://dx.doi.org/10.6035/Dossiers.2017.22.3 
HITE, Shere (2000): Sex and business, Financial Times Prentice Hall.

KEMP, Sandra y SQUIRES, Judith (1998): Feminisms (Oxford Readers), Oxford,Oxford University Press

LYNCH, Edith M. (1973): Executive Suite: Feminine Style, Nueva York, AMACOM.

Martin, Michèle (1991): «Hello, Centrale?: Gender, Technology, and Culture in the Formation of Telephone Systems, McGill-Queen's Press.

Mikman, Ruth (1987): Gender at Work: The Dynamics of Job Segregation by Sex During World War II, Chicago, University of Illinois Press.

Millar, Melanie (1998): Cracking the Gender Code: Who Rules the Wired World?, Canadian Scholars' Press.

PLANT, Sadie (1998): Zeros and Ones: Digital Women and the New Technoculture, London, Fourth Estate.

SANDBerg, Sheryl (2013): Lean In: Women, Work, and the Will to Lead, Nueva York, Knopf Doubleday Publishing Group.

Suchman, Lucy (2008): Feminist STS and the Sciences of the Artificial. The Handbook of Science and Technology Studies, Cambridge, MIT Press.

TAYLOR, Stephen John y BOGDAN, Robert (1985): Introducción a los métodos cualitativos. Barcelona, Buenos Aires, México, Paidós.

Tyson, Laura D'Andrea (2003): The Tyson Report on the Recruitment and Development of NonExecutive Directors, London, London Business School.

WaJCMAN, Judy (1998): Managing like a Man, Cambridge, Polity Press.

WAICMAN, Judy (2009): Feminist theories of technology, Cambridge, Journal of Economics.

WeBSTER, Juliet (2005): Why are women still so few in IT? The gender politics of IT, Middlesex, University Press. 